\title{
CR4056: A NOVEL POTENT ANTI-NOCICEPTIVE AGENT FOR SEVERAL ANIMAL MODELS OF NEUROPATHIC PAIN
}

Meregalli C. ${ }^{a}$, Chiorazzi A. ${ }^{a}$, Canta A. ${ }^{a}$, Carozzi V. ${ }^{a}$, Oggioni N. ${ }^{a}$, Ferrari F $^{\mathrm{b}}$., Lanza M. ${ }^{\mathrm{b}}$, Letari O. ${ }^{\mathrm{b}}$, Caselli G. ${ }^{\mathrm{b}}$, Costa B. $^{\mathrm{c}}$, Cavaletti G. ${ }^{\mathrm{a}}$, Tredici $\mathrm{G}^{\mathrm{a}}$.

a) Department of Neuroscience and Biomedical Technologies, University of Milan-Bicocca, Monza, ITALY

b) Pharmacology \& Toxicology Department, Rottapharm|Madaus Research Center, Monza (MI), ITALY

c) Department of Biotechnology and Bioscience, University of Milano-Bicocca, Milan, ITALY

Introduction: CR4056 is a novel imidazoline-2 receptor ligand endowed with low affinity MAO inhibition properties.

Objectives: Aims of this study was to test with different methods the anti-nociceptive effect of CR4056 in rat models of neuropathic pain, i.e. Bortezomib(Btz)-induced neuropathic pain, streptozotocin-induced diabetes and chronic constriction injury (CCI).

Methods: In the Btz model $(0.20 \mathrm{mg} / \mathrm{kg} 3 \mathrm{qwx} 8 \mathrm{iv})$ and in the experimental diabetes model CR4056 was administered at $6-60 \mathrm{mg} / \mathrm{kg}$ po. In the CCI mononeuropathy model CR4056 was administered at 10-80 mg/kg po. Nerve conduction velocity (NCV), dynamic, Randall-Selitto, plantar and von Frey tests were used to assess the effect of CR4056.

Results: After 8 weeks of treatment, Btz induced a severe reduction in NCV and this impairment was not reversed by CR4056. The behavioral assessment showed a significant mechanical allodynia in rats treated wit Btz for 8 wks and the daily administration of CR4056 significantly reduced this condition. This effect was persistent along the entire period of administration after Btz withdrawal (2 weeks).

In the models of streptozotocin-induced diabetes and CCI, treatment with CR4056 significantly attenuated mechanical and thermal hyperalgesia, while CR4056 had no effect on mechanical allodynia in both experimental models.

Conclusions: These results promote the use of CR4056 as a potential treatment in the chronic neuropathic pain therapy. 